\title{
The Risk of Nosocomial Infection with Multidrug-Resistant Corynebacterium urealyticum After Prostate Cancer Surgery
}

\author{
Zohre Baseri ${ }^{1}$, Mohammad Shayestehpour ${ }^{2,3}$, Yousef Erfani ${ }^{4}$, Arezoo Rasti ${ }^{5}$, Bizhan Romani ${ }^{6,7}$, \\ Behrooz Mozaffari Namin ${ }^{8}$, Abbas Ahmadi Vasmehjani ${ }^{9}$, Kaveh Sadeghi ${ }^{9}$ and Sajad Yaghoubi ${ }^{10,}{ }^{*}$ \\ ${ }^{1}$ Department of Microbiology, Faculty of Medicine, Iran University of Medical Sciences, Tehran, Iran \\ ${ }^{2}$ Autoimmune Diseases Research Center, Kashan University of Medical Sciences, Kashan, Iran \\ ${ }^{3}$ Department of Microbiology and Immunology, Faculty of Medicine, Kashan University of Medical Sciences, Kashan, Iran \\ ${ }^{4}$ Department of Medical Laboratory Sciences, School of Allied Medical Sciences, Tehran University of Medical Sciences, Tehran, Iran \\ ${ }^{5}$ Oncopathology Research Centre, Iran University Medical Sciences, Tehran, Iran \\ ${ }^{6}$ Department of Biochemistry, University of Alberta, Edmonton, Alberta, T6G 2E1, Canada \\ ${ }^{7}$ Cellular and Molecular Research Center (CMRC), Faculty of Medicine, Ahvaz Jundishapur University of Medical Sciences (AJUMS), Ahvaz, Iran \\ ${ }^{8}$ Department of Microbiology of Pathobiology, School of Public Health, Tehran University of Medical Sciences, International Campus, Tehran, Iran \\ ${ }^{9}$ Virology Department, School of Public Health, Tehran University of Medical Sciences, Tehran, Iran \\ ${ }^{10}$ Department of Clinical Microbiology, Iranshahr University of Medical Sciences, Iranshahr, Iran \\ "Corresponding author: Department of Clinical Microbiology, Iranshahr University of Medical Sciences, Iranshahr, Iran. Tel: +98-9361620319, Fax: +98-2188954813, Email: \\ shayesteh2009@yahoo.co.uk
}

Received 2017 December 19; Revised 2018 September 04; Accepted 2018 September 29.

\begin{abstract}
Corynebacterium urealyticum is a Gram-positive, lipophilic, multidrug resistant, and urease positive microorganism with diphtheroid morphology. C. urealyticum causes several diseases such as urinary tract infection, chronic urological disease, urinary tract infections, and bacteremia in immunocompromised individuals. This study reports a rare case with nosocomial infection and hematuria caused by multidrug-resistant $C$. urealyticum after prostate cancer surgery.
\end{abstract}

Keywords: Corynebacterium urealyticum, Urinary Tract Infections, Prostate Cancer

\section{Introduction}

Corynebacterium urealyticum (known as coryneform CDC 79 group D2) is a Gram-positive, slow-growing, multidrug-resistant, and urease positive microorganism with a potent urease activity (1). C. urealyticum, as an opportunistic nosocomial pathogen, causes various diseases such as acute cystitis, pyelonephritis, encrusted cystitis, encrusted pyelitis, and alkaline encrusted cystitis. Infection with $C$. urealyticum mostly occurs in patients with chronic urological diseases and sometimes leads to bacteremia $(2,3)$. Its strong urease activity results in prominent urinary tract infections (UTIs) (4). Additionally, it causes infrequent non-urinary infections such as pneumonia, peritonitis, cardiac valve infection, osteomyelitis, postoperative wounds, and soft tissue necrosis (5). Infection by C. urealyticum is associated with several risk factors, including prolonged hospitalization, immunodeficiency diseases, urological procedures, and broad-spectrum antibiotic therapy $(1,6,7)$. We report a rare case of an 80-yearold Iranian man who had developed nosocomial infection and hematuria associated with multidrug-resistant C. ure- alyticum after prostate cancer surgery.

\section{Case Presentation}

An 80-year-old Iranian man, who was diagnosed with prostate cancer, was subjected to prostatectomy whose prostate was removed and he was discharged. He was accidentally fallen at home 4 days after the operation that resulted in a femur fracture. He was then hospitalized for hip surgery. He suffered from leg edema, hematuria, and dysuria after 2 days of hospitalization. Initial symptoms included chills and rapid breathing. Primary diagnosis was venous thrombus in the deep veins of the leg(DVT) and clot in the lungs due to pulmonary embolism. In the clinical examinations, blood pressure of $98 / 70 \mathrm{mmHg}$, heart rate of 72 beats/min, respiratory rate of 33 breaths/min, and body temperature of $38^{\circ} \mathrm{C}$ were recorded. The prostate-specific antigen (PSA) level, liver enzymes, and CT chest were normal.

Treatment started with antibiotics, including imipenem, ceftriaxone, and ciprofloxacin. He also had 
acute hematuria because of prostatectomy in addition to receiving chemotherapy and hormone therapy. In the emergency room, fever and the temporary loss of consciousness associated with hematuria and acute aggravation of chronic renal failure were considered due to the relapse of the prostate cancer. Meanwhile, his temperature was increasing with hematuria. The results of urine and blood tests are shown in Table 1 . Urine culture yielded 100,000 colonies/mL of Gram-positive bacilli after 4 days of incubation, which was identified as C. urealyticum.

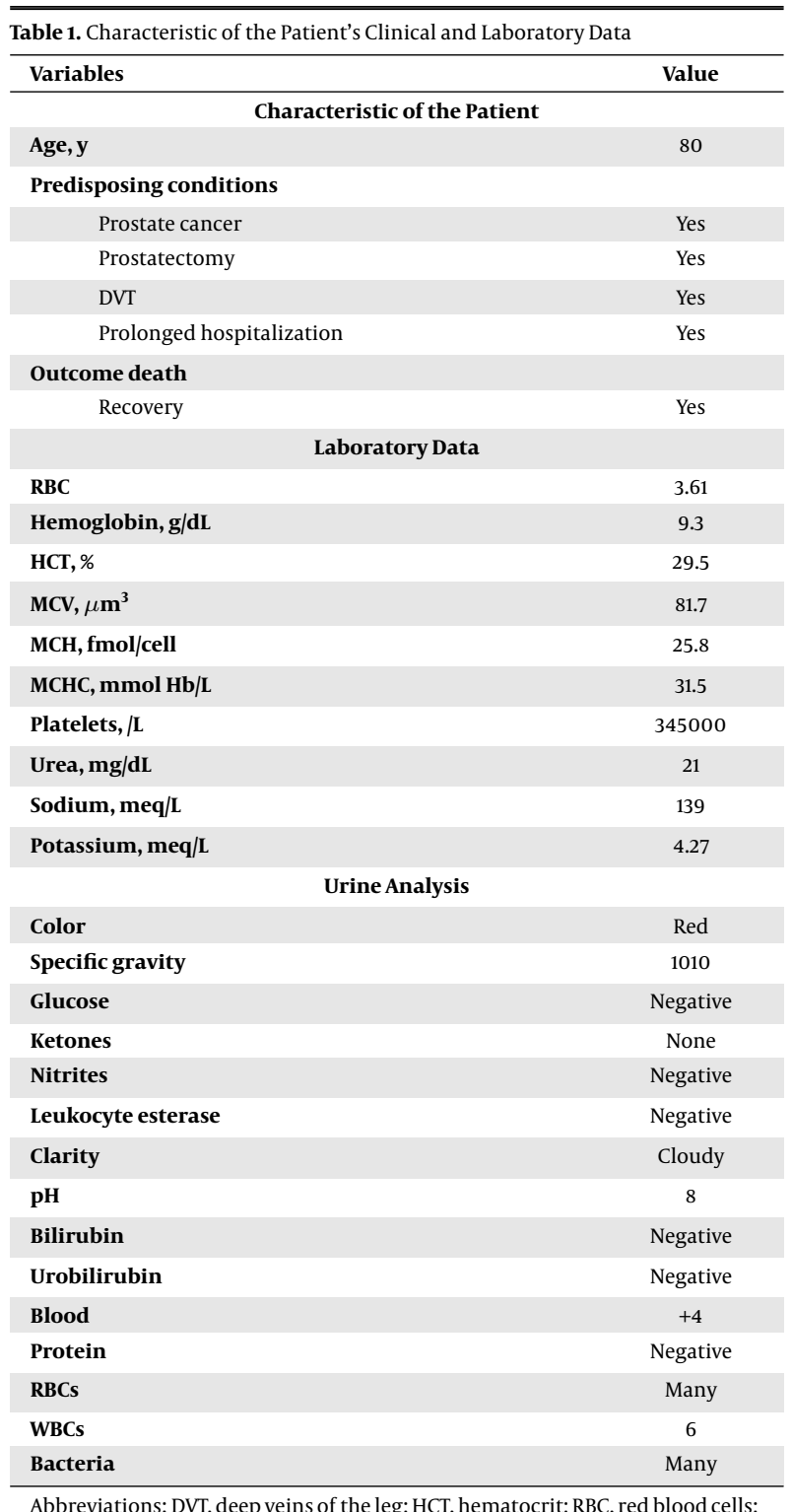

Abbreviations: DVT, deep veins of the leg; HCT, hematocrit; RBC, red blood cells; WBC, White blood cells.

Antimicrobial susceptibility test was conducted by disc diffusion method on Muller Hinton blood agar and broth microdilution with vancomycin, teicoplanin, ampicillin (10 mg), cephalothin (30 mg), erythromycin (15 $\mathrm{mg}$ ), gentamicin, imipenem (10 mg), tetracycline (30 mg), rifampicin (5 mg), ciprofloxacin (5 $\mathrm{mg}$ ), and ofloxacin (5 mg) (8). Results showed that the bacterial isolate was resistant to ceftriaxone, erythromycin, rifampicin, ciprofloxacin, imipenem, and co-trimoxazole but sensitive to vancomycin and teicoplanin. Vancomycin was prescribed for 10 days and the fever subsided gradually and the patient was then discharged. There was no recurrence of bacteremia and hematuria during the follow-up. Written informed consent was obtained from the patient.

\section{Discussion}

Corynebacterium urealyticum is an emerging multidrugresistant, potentially pathogenic microorganism that causes nosocomial and urinary tract infections in patients with advanced and severe urological disorders. It causes different diseases, which arise from prolonged use of a urinary catheter, long periods of hospitalization, compromised immune system, kidney transplantation, urological procedure, chronic debilitating disease, broad-spectrum antibiotic treatment, and cytotoxic drug usage (9). Our results suggest that in addition to the aforementioned factors, prostatectomy in prostate cancer should also be considered one of the risk factors for contracting the infection by C. urealyticum.

This patient had a long-term recovery for over 10 days, which could be due to an improper choice of antibiotics (ceftriaxone and imipenem) until he took vancomycin and then his recovery was completed. Consistent with our report, there was a patient who was treated by vancomycin (1). In most cases, the majority of patients receiving sufficient doses of antibiotics are cured, whereas only a few cases of $C$. urealyticum (mostly with acute cystitis) are not easily cured even when treated with multiple antibiotics. Glycopeptides, mainly vancomycin, tetracycline, and fluoroquinolones are the best drugs of choice in the treatment and have been used with great effects on many patients with UTIs (5). A previous study reported 6 bacteremic patients infected with multidrug-resistant strains of $C$. urealyticum. The patients were cured with an antibiotic, such as vancomycin that was in vitro active against $C$. urealyticum (10). In agreement with our study, the results of several studies emphasize the emergence of multidrugresistant $C$. urealyticum with susceptibility to vancomycin and teicoplanin as the drugs of choice.

Based on MIC results, most antibiotics are effective in chronic, device-related, urological infections (10). A number of studies have shown that patients with isolated multidrug-resistant $C$. urealyticum are treated by van- 
comycin and teicoplanin (8). Many strains of C. urealyticum have been shown to be resistant to ofloxacin, norfloxacin, and ciprofloxacin as we found in the current study. Salem et al. (1) demonstrated that only $20.3 \%$ of C. urealyticum isolates were susceptible to ciprofloxacin. Our results showed that our isolate was resistant to ciprofloxacin as well as ceftriaxone. As a result, our recommendation is to stop their prescription in multidrug-resistant bacteria. Surprisingly, we then found other patients in the ward infected by C. urealyticum proposing the ability of the microorganism for cross-contamination and transmission. This highlighted the importance of non-diphtheria corynebacterium species in hospital infections, particularly for clinicians and microbiologists. Thus accurate identification of clinical isolates is important to determine the role of nondiphtheria corynebacterium. It is noteworthy that presenting the sign of UTIs, such as high urine $\mathrm{pH}$, bladder stones, recent urological surgeries, negative routine urine culture, aging, and antibiotic resistance found in UTIs may indicate the infection of $C$. urealyticum. In order to inhibit bacterial transmission from infected patients, hand washing, using gloves, and avoiding unnecessary catheterization should be applied. Diagnostic laboratories should not ignore diphtheroid shaped microorganisms, particularly in pure cultures of elderly and immunocompromised patient samples (10).

These bacteria should be considered common etiological causes of nosocomial infections. In conclusion, nosocomial infections are usually associated with high morbidity, mortality, and financial burden. C. urealyticum is an opportunistic pathogen, causing different diseases, particularly UTIs (11). Sufficient knowledge of emerging pathogens and their resistance profile are critical for the treatment of nosocomial infections $(12,13)$.

\section{Footnotes}

Conflict of Interests: All contributing authors declare no conflict of interest.

Funding/Support: No funding was provided for this research.

Patient Consent: Written informed consent was obtained from the patient.

\section{References}

1. Salem N, Salem L, Saber S, Ismail G, Bluth MH. Corynebacterium urealyticum: A comprehensive review of an understated organism. Infect Drug Resist. 2015;8:129-45. doi: 10.2147/IDR.S74795. [PubMed: 26056481]. [PubMed Central: PMC4445954].

2. Chung CS, Liao CH, Cheng SL, Lin TH, Hsueh PR. Percutaneous nephrostomy tube-associated bacteremia caused by Corynebacterium urealyticum. J Microbiol Immunol Infect. 2008;41(6):525-7. [PubMed: 19255698].

3. Soriano F, Huelves L, Naves P, Rodriguez-Cerrato V, del Prado G, Ruiz V, et al. In vitro activity of ciprofloxacin, moxifloxacin, vancomycin and erythromycin against planktonic and biofilm forms of Corynebacterium urealyticum. J Antimicrob Chemother. 2009;63(2):353-6. doi: 10.1093/jac/dkn491. [PubMed: 19056748].

4. Lopez-Medrano F, Garcia-Bravo M, Morales JM, Andres A, San Juan R, Lizasoain $\mathrm{M}$, et al. Urinary tract infection due to Corynebacterium urealyticum in kidney transplant recipients: An underdiagnosed etiology for obstructive uropathy and graft dysfunction-results of a prospective cohort study. Clin Infect Dis. 2008;46(6):825-30. doi: 10.1086/528713. [PubMed: 18260786].

5. Wood CA, Pepe R. Bacteremia in a patient with non-urinary-tract infection due to Corynebacterium urealyticum. Clin Infect Dis. 1994;19(2):367-8. [PubMed: 7986931].

6. Soriano F, Tauch A. Microbiological and clinical features of Corynebacterium urealyticum: Urinary tract stones and genomics as the Rosetta Stone. Clin Microbiol Infect. 2008;14(7):632-43. doi: 10.1111/j.1469-0691.2008.02023.x. [PubMed:18558935].

7. Soriano F, Zapardiel J, Nieto E. Antimicrobial susceptibilities of Corynebacterium species and other non-spore-forming grampositive bacilli to 18 antimicrobial agents. Antimicrob Agents Chemother. 1995;39(1):208-14. [PubMed: 7695308]. [PubMed Central: PMC162510].

8. Kiehlbauch JA, Hannett GE, Salfinger M, Archinal W, Monserrat C, Carlyn C. Use of the National Committee for Clinical Laboratory Standards guidelines for disk diffusion susceptibility testing in New York state laboratories. J Clin Microbiol. 2000;38(9):3341-8. [PubMed: 10970381]. [PubMed Central: PMC87384].

9. Munoz-Bellido JL, Munoz-Criado S, Garcia-Rodriguez JA. In-vitro activity of psychiatric drugs against Corynebacterium urealyticum (Corynebacterium group D2). J Antimicrob Chemother. 1996;37(5):1005-9. [PubMed: 8737151].

10. Fernandez-Natal I, Guerra J, Alcoba M, Cachon F, Soriano F. Bacteremia caused by multiply resistant corynebacterium urealyticum: Six case reports and review. Eur J Clin Microbiol Infect Dis. 2001;20(7):514-7. [PubMed: 11561814].

11. Mohammad Nejad A, Shayestehpour M, Romani B, Mozaffari Namin B, Ahmadi Vasmehjani A, Yaghoubi S. A rare case of pneumococcal and meningococcal mixed meningitis in an infant with cardiac dysfunction. Arch Clin Infect Dis. 2018;13(6). e65270.

12. Beiranvand M, Amin M, Hashemi-Shahraki A, Romani B, Yaghoubi S, Sadeghi P. Antimicrobial activity of endophytic bacterial populations isolated from medical plants of Iran. Iran J Microbiol. 2017;9(1):11-18. [PubMed Central: PMC5533999].

13. Saeidi Y, Pournajaf A, Gholami M, Hasannejad-Bibalan M, Yaghoubi $S$, Khodabandeh M. Determination of Helicobacter pylori virulenceassociated genes in duodenal ulcer and gastric biopsies. Med J Islam Repub Iran. 2017;31(95). [PubMed Central: PMC6014795]. 